\title{
Interaction of stromal and microvascular components in keratocystic odontogenic tumors
}

\author{
Ernesto Santos Sousa-Neto ${ }^{1}$, Maria Cristina Teixeira Cangussu ${ }^{1,2}$, Clarissa Araújo Gurgel ${ }^{1,3,4}$, Vanessa \\ Sousa Guimarães ${ }^{4}$, Eduardo Antônio Gonçalves Ramos ${ }^{3}$, Flávia Caló Aquino Xavier ${ }^{1,5}$, Patrícia Ramos \\ Cury $^{1,6}$, Braúlio Carneiro Júnior ${ }^{1,7}$, Rosalia Leonardi ${ }^{8}$, Jean Nunes Dos Santos ${ }^{1,3,5}$ \\ ${ }^{1}$ Dentistry and Health Postgraduate Program, School of Dentistry, Federal University of Bahia, Salvador, Bahia, Brazil; ${ }^{2}$ Department of \\ Dental Public Health, School of Dentistry, Federal University of Bahia Salvador, Bahia, Brazil; ${ }^{3}$ Human Pathology Postgraduate \\ Program, Oswaldo Cruz Foundation, Fiocruz, Salvador, Bahia, Brazil; ${ }^{4}$ Laboratory of Pathology and Molecular Biology, Oswaldo Cruz \\ Foundation, Fiocruz, Salvador, Bahia, Brazil; ${ }^{5}$ Laboratory of Oral Surgical Pathology, School of Dentistry, Federal University of Bahia, \\ Salvador, Bahia, Brazil; ${ }^{6}$ Division of Periodontics, School of Dentistry, Federal University of Bahia, Salvador, Bahia, Brazil; ${ }^{7}$ Department \\ of Oral and Maxillofacial Surgery, School of Dentistry, Federal University of Bahia, Salvador, Bahia, Brazil; ${ }^{8}$ Department of Medical and \\ Surgical Sciences, University of Catania, Catania, Italy
}

OBJECTIVE: Little is known about the interaction of stromal components in odontogenic tumors. Thus, the aim of this study was to investigate mast cells (MCs), myofibroblasts, macrophages, and their possible association with angiogenesis and lymphangiogenesis in keratocystic odontogenic tumors (KCOTs).

MATERIAL AND METHODS: Thirty cases of KCOTs were included and analyzed by immunohistochemistry for mast cell tryptase, $\alpha$-SMA, CD34, CDI63, and D240. For comparative purpose, 15 radicular cysts (CRs) and 7 pericoronal follicles (PFs) were included.

RESULTS: There was an increase in MCs for RCs and this difference was significant when they were compared to KCOTS and PFs. A significant increase in the density of MFs was observed for KCOTs when compared to RCs and PFs $(P=0.00)$. No significant difference in CDI63positive macrophages $(P=0.084)$ and CD34-positive vessels $(P=0.244)$ densities was observed between KCOTs, RCs, and PFs, although KCOTs showed a higher density of all proteins. Significant difference in lymphatic vessel density was observed for KCOTs when compared to RCs and PFs $(P=0.00)$. Positive correlation was observed between mast cell tryptase and CD34 in KCOTs $(P=0.025)$.

CONCLUSIONS: A significant interaction between the MC population and CD34-positive vessels in KCOTs supported the hypothesis that MCs and blood vessels contribute to the stromal scaffold of KCOT.

J Oral Pathol Med (2016) 45: 557-564

Correspondence: Jean Nunes dos Santos, Av. Araújo Pinho, 62, Canela, Salvador, Bahia 40110150, Brazil. Tel: 55713283 9019, Fax: 55718962 , E-mail: jeanunes@ufba.br

Accepted for publication November 14, 2015
Keywords: angiogenesis; cells; keratocyst; lymphangiogenesis; macrophages; myofibroblasts

\section{Introduction}

Keratocystic odontogenic tumors (KCOTs) arise from epithelial remnants of the dental lamina. In view of their clinical, pathological and molecular characteristics, especially when compared to other developmental odontogenic cysts (1-3), the World Health Organization (WHO) adopted the term keratocystic odontogenic tumor (1). Furthermore, the invasion capacity, aggressive nature, and destructive growth of KCOT are related to unknown biological factors of the stroma and parenchyma, which confer a distinct biological behavior to this tumor $(1,3,4)$.

The tumor microenvironment is composed of a complex variety of stromal components including cells and vessels that exert different regulatory functions favoring tumor growth. Mast cells are important cells of the innate and acquired immune system (5) that secrete a range of biologically active products stored in their cytoplasmic granules including tryptase and chymase $(6,7)$. These cells also participate in the process of angiogenesis and in tissue repair and remodeling (8-10). According to Ribatti and Crivellato (5) and Stockmann et al. (10), MCs are involved in tumorigenesis and tumor progression, mainly as a result of their capacity to release proangiogenic factors.

Myofibroblasts (MFs) are specialized cells that exhibit features of fibroblasts and smooth muscle cells $(8,11)$. They possess contractile capacity and are able to synthesize different extracellular matrix components such as collagen types I and II $(8,12)$. MFs have been associated with the progression and development of different odontogenic cysts and tumors, including KCOT (13-16). Nevertheless, some 
authors suggest that tryptase released by MCs induces the proliferation of fibroblasts and their transdifferentiation into MFs (12).

Another type of cell found in the tumor microenvironment are macrophages (17-19). Although several studies have shown that macrophages can destroy tumor cells in vitro, it is currently accepted that these cells also participate in the promotion of tumor growth (17-19). Tumor-associated macrophages are derived from blood monocytes, which differentiate into mature macrophages in the tumor microenvironment (17-19). This cell population shows specialized functional properties, inducing M1- and M2-polarized macrophages which in turn, may or may not promote the progression of cancer (17).

Angiogenesis contributes to the invasion potential and aggressiveness of a tumor as well as tumor initiation as this process contributes to the growth of tumor cells (20-23). Angiogenesis is a complex process that is regulated by different intercellular signaling pathways that exert both proangiogenic and anti-angiogenic functions (20-23). Despite the large number of studies on angiogenesis as a mechanism of tumor growth, little is known about the mechanisms involved in the formation of lymphatic vessels, a process called lymphangiogenesis (23). The identification of the phenomenon of lymphangiogenesis in the tumor stroma can also serve as a basis to evaluate the aggressive potential of tumors (23).

MCs can influence the differentiation/transdifferentiation of fibroblasts into MFs (12) and these cells are found in odontogenic cysts and tumors (4, 13-16, 24, 25). In addition, the presence of macrophages, angiogenesis, and lymphangiogenesis are related to tumor development (17, 26-30), also including odontogenic tumors and cysts (15, 30-34). To our knowledge, there are no studies focusing on the interaction of these stromal components nor on the role of MCs in the myofibroblastic differentiation in KOCTs. Therefore, the objective of the present study was to investigate the role of these cell components and their possible association with the processes of angiogenesis and lymphangiogenesis in a series of KCOTs. Radicular cysts (CRs) and pericoronal follicles (PFs) were also included in the study for comparison.

\section{Material and methods}

After approval by the Ethics Committee of the School of Dentistry, Federal University of Bahia, Salvador, Bahia, 30 cases of sporadic KCOTs diagnosed between 2008 and 2014 were included in this study. The cases were retrieved from the archives of the Laboratory of Surgical Pathology, School of Dentistry, Federal University of Bahia, Salvador. The histopathological diagnosis was revised and classified by an experienced pathologist (J.N.S.) based on the World Health Organization classification (1). Fifteen cases of RC and seven PFs of impacted third molars were also included.

For immunohistochemistry, $3-\mu \mathrm{m}$-thick histological sections were obtained from $10 \%$ formalin-fixed and paraffinembedded material. The sections were deparaffinized and rehydrated. The EnVision ${ }^{\mathrm{TM}}$ polymeric system was used for immunostaining. Table 1 shows the specificity, clone, antigen retrieval solution, dilution, and time of incubation for the antibodies used. After antigen retrieval, endogenous peroxidase was blocked by immersion of tissue sections in a solution of $3 \%$ hydrogen peroxide and distilled water for $10 \mathrm{~min}$, protected from light. The antibodies were diluted with background-reducing solution (DAKO Corporation, Carpinteria, USA). The sections were then incubated with the primary antibodies in room temperature for $1 \mathrm{~h}$. After, the sections were immersed in a solution of $1 \%$ Tris- $\mathrm{HCl} /$ $\mathrm{BSA}$, Tris- $\mathrm{HCl} /$ Triton, and distilled water for $5 \mathrm{~min}$ each. Next, non-specific background staining was blocked using Protein Block (DAKO Corporation, Carpinteria, USA) for 10 min.

The reaction was developed with 3,3'- diaminobenzidine (DAKO Corporation, Carpinteria, USA) in a dark chamber for $5 \mathrm{~min}$ and counterstained with Harris hematoxylin for $15 \mathrm{~s}$.

The positive controls of the reactions consisted of pyogenic granuloma tissues with known positivity for these antibodies. Sections in which the primary antibodies were replaced with non-immune serum served as negative controls.

Each case was analyzed by two previously trained examiners under a high-definition light microscope (Axiostar Plus, Zeiss, Germany, 2008) in up to 10 fields at $400 \times$ magnification. The images were captured with a digital camera (AxioCam ICc3, Zeiss, Germany, 2008) and transferred to a computer system.

MCs that exhibited a brown staining were defined as positive. Additionally, the presence of degranulation was also characterized. Mast cell tryptase-positive cells were quantified by calculating the mean number in each case. The density of MCs was then calculated by dividing the mean

Table 1 Antibodies used in immunohistochemical analysis

\begin{tabular}{|c|c|c|c|c|c|}
\hline Specificity & Clone & Antigen retrieval & Dilution & Time and temperature of incubation & Manufacturer \\
\hline Mast cell tryptase & AA1 & $1 \%$ trypsin at $37^{\circ} \mathrm{C}$ & $1: 50$ & $60 \mathrm{~min} / \mathrm{RT}$ & Dako $^{a}$ \\
\hline$\alpha-\mathrm{SMA}$ & $1 \mathrm{~A} 4$ & $\begin{array}{l}\text { Citrate, } \mathrm{pH} 6.0, \\
\text { at } 98^{\circ} \mathrm{C}\end{array}$ & $1: 150$ & $60 \mathrm{~min} / \mathrm{RT}$ & Dako $^{\mathrm{a}}$ \\
\hline CD163 & $\mathrm{GHI} / 61$ & $\begin{array}{l}\text { Citrate, } \mathrm{pH} 6.0 \text {, } \\
\text { at } 98^{\circ} \mathrm{C}\end{array}$ & $1: 100$ & 18 h/overnight/RT & Santa $\mathrm{Cruz}^{\mathrm{b}}$ \\
\hline CD34 & QBEND 10 & $\begin{array}{l}\text { Citrate, } \mathrm{pH} 6.0, \\
\text { at } 98^{\circ} \mathrm{C}\end{array}$ & $1: 50$ & $60 \mathrm{~min} / \mathrm{RT}$ & Dako $^{\mathrm{a}}$ \\
\hline Anti-podoplanin & D240 & $\begin{array}{l}\text { Citrate, } \mathrm{pH} 6.0, \\
\text { at } 98^{\circ} \mathrm{C}\end{array}$ & $1: 200$ & $45 \mathrm{~min} / \mathrm{RT}$ & Dako $^{\mathrm{a}}$ \\
\hline
\end{tabular}

a Dako Corporation, Carpinteria, USA; ${ }^{\mathrm{b}}$ Santa Cruz, California, USA, RT: room temperature. 
number of cells by the area analyzed, obtaining the mean density of MCs per $\mathrm{mm}^{2}$ (4). $\alpha$-SMA-positive cells located at the periphery of blood vessels were excluded.

MFs were identified exhibiting a brown staining. The values obtained for each field were summed and the total number of anti- $\alpha$-SMA-positive cells was established. The latter was used to calculate the mean density of anti- $\alpha$-SMApositive cells per $\mathrm{mm}^{2}(11)$.

For the evaluation of macrophages using the anti-CD163 antibody, the same method as described for MC count was used.

Blood vessels were identified using CD34 and those cases in which endothelial cells exhibited brown staining were defined as positive. The angiogenic index was established by determining microvessel density (MVD) as described by Maeda et al. (27). The values obtained for each field were summed and the total number of microvessels was established, and microvessel density per $\mathrm{mm}^{2}$ was obtained. Isolated immunopositive cells and groups of immunopositive cells were also considered microvessels, irrespective of the presence of a conspicuous lumen (20). Additionally, large-caliber vessels with a lumen greater than $50 \mu \mathrm{m}$ were excluded.

Cases in which lymphatic endothelial vessels exhibited brown staining were defined as positive. The lymphangiogenic index was established by determining the lymphatic microvessel density (LMVD) per $\mathrm{mm}^{2}$ using the method described by Maeda et al. (27) and adapted for analysis of this marker.

The presence of an inflammatory infiltrate in KCOTs was also evaluated and classified as non-significant inflammation and significant inflammation (4).

The results were reported as mean density $/ \mathrm{mm}^{2} \pm$ standard deviation. Statistical analysis was performed using the Minitab program for Windows, version 14 [Minitab, Inc., USA]. One-way ANOVA with Tukey's post-test correction was used for the comparison of markers according to the type of lesion. The correlation between markers within the same lesion was evaluated using Pearson's adjusted correlation coefficient. The Student's $t$-test was used to evaluate the association between the markers and presence of inflammation. A level of significance of $5 \%$ was adopted $(P<0.05)$.

\section{Results}

\section{Immunoexpression of mast cells}

Mast cells were identified in all KCOT specimens mainly in the stromal tissue with or without inflammation (Fig. 1A). However, they were decreased in relation to RCs, except PFs. These cells were also present in the epithelial lining, (Fig. 2A), observed in $12.2 \%(n=4)$ of cases. In addition to the presence of MCs containing brown cytoplasmic granules, these granules were also detected scattered around stained cells, a finding suggesting degranulation.

Mast cells were detected in all $15 \mathrm{RC}$ specimens. These cells were also found in the deep and superficial regions of the fibrous capsule, and the highest concentration was observed near areas of inflammation (Fig. 1B). In three cases, MCs were observed in the cyst lining and most of them showed these cells within the cystic lumen (Fig. 2B).
Mast cells were also within and surrounding odontogenic epithelial remnants (Fig. 1B) and showed degranulation.

In PFs, MCs were detected in only 4 cases $(57.14 \%)$ and their spatial distribution was similar to that seen in KCOTs and RCs (Fig. 1C). However, there was a reduction in MCs for KOCTs in relation to RCs and an increase in relation to PFs. A significant difference of MC density between RCs and PFs and KCOTs $(P<0.01$, one-way ANOVA), as well as between KCOTs and PFs ( $P=0.00$, one-way ANOVA), was found (Table 2)

\section{Immunoexpression of $\alpha-S M A$}

All KCOT specimens analyzed $(n=30)$ were immunopositive for $\alpha$-SMA. However, there was an increase in MCs for KCOTs in relation to RCs and PFs. These cells were located in the stroma, most of the time as single cells, arranged or not parallel to the epithelial lining (Fig. 1D). No MFs were detected in the stroma in areas where the epithelial lining was absent. Myofibroblasts were sometimes observed in areas of granulation tissue (Fig. 2C), although in most cases they were detected in areas where inflammation was absent.

RCs revealed the presence of MFs in $100 \%$ of the cases analyzed $(n=15)$. Myofibroblasts exhibited different shapes in the presence of granulation tissue (Figs 1E, 2D). In PFs, anti- $\alpha$-SMA immunostaining showed the presence of MFs in all cases analyzed $(n=7)$ (Fig. 1F). A significant difference in the density of MFs was observed for KCOTs when compared to RCs and PFs $(P<0.01$, one-way ANOVA), but not between RCs and PFs $(P>0.05$, oneway ANOVA, Table 2).

\section{Immunoexpression of CD163}

CD163-positive macrophages were detected in $73.33 \%$ $(n=22)$ of KCOT cases and were increased in relation to RCs and PFs. In most cases, these cells contained brown granules. Macrophages were distributed beneath the epithelial lining, and particularly in deeper regions of the fibrous capsule (Fig. 1G) especially when the inflammation was significant. Macrophages were detected in the tumor epithelium in $6.6 \%(n=2)$ of cases (Fig. 2E).

In RCs, positive immunostaining for CD163 was observed in $80 \%(n=12)$ of the cases analyzed (Fig. 1H). CD163-positive macrophages were not detected in three specimens $(20 \%)$. Macrophages were observed in superficial and deep areas, irrespective of the presence or absence of inflammation.

Positive immunostaining for CD163 was observed in $71.42 \%(n=5)$ of the PF cases analyzed, but this cell population was rare (Fig. 1I). No macrophages were detected in two specimens (28.58\%). No statistically significant difference in macrophage density was observed between KCOTs, RCs and PFs $(P=0.084$, one-way ANOVA, Table 2).

\section{Immunoexpression of CD34}

CD34-positive blood vessels were detected in $100 \%$ of KCOT cases (Fig. 1J) and were increased in relation to RCs and PFs. The mean angiogenic indices in KCOT ranged from 5.52 to 27.3 (mean $16.58 \pm 5.98$ ). CD34-positive blood vessels were observed in $100 \%$ of the RC specimens 
RADICULAR CYST
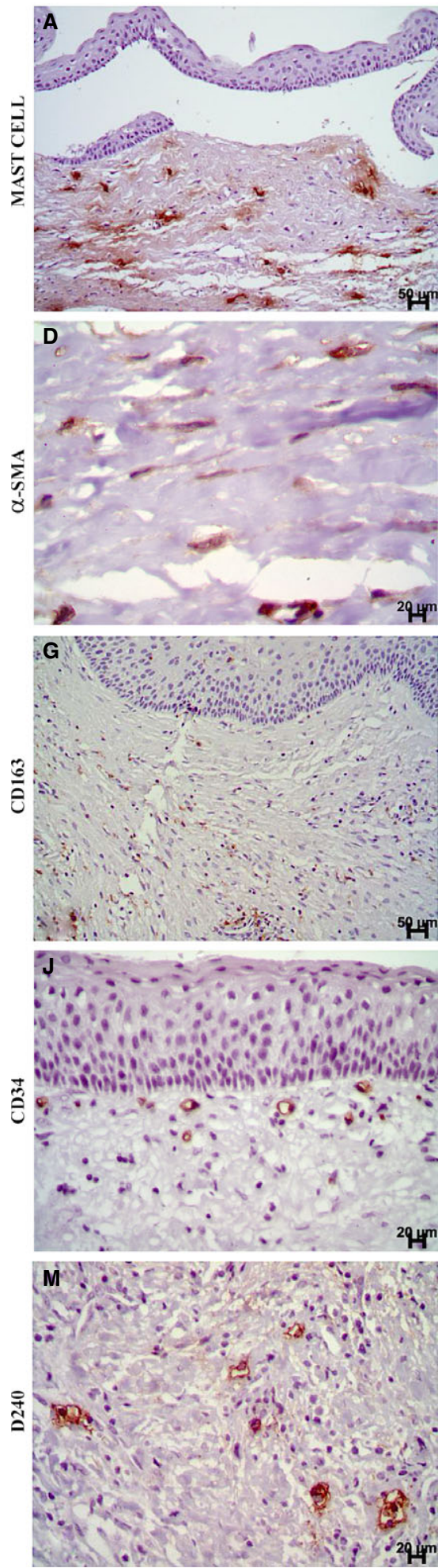
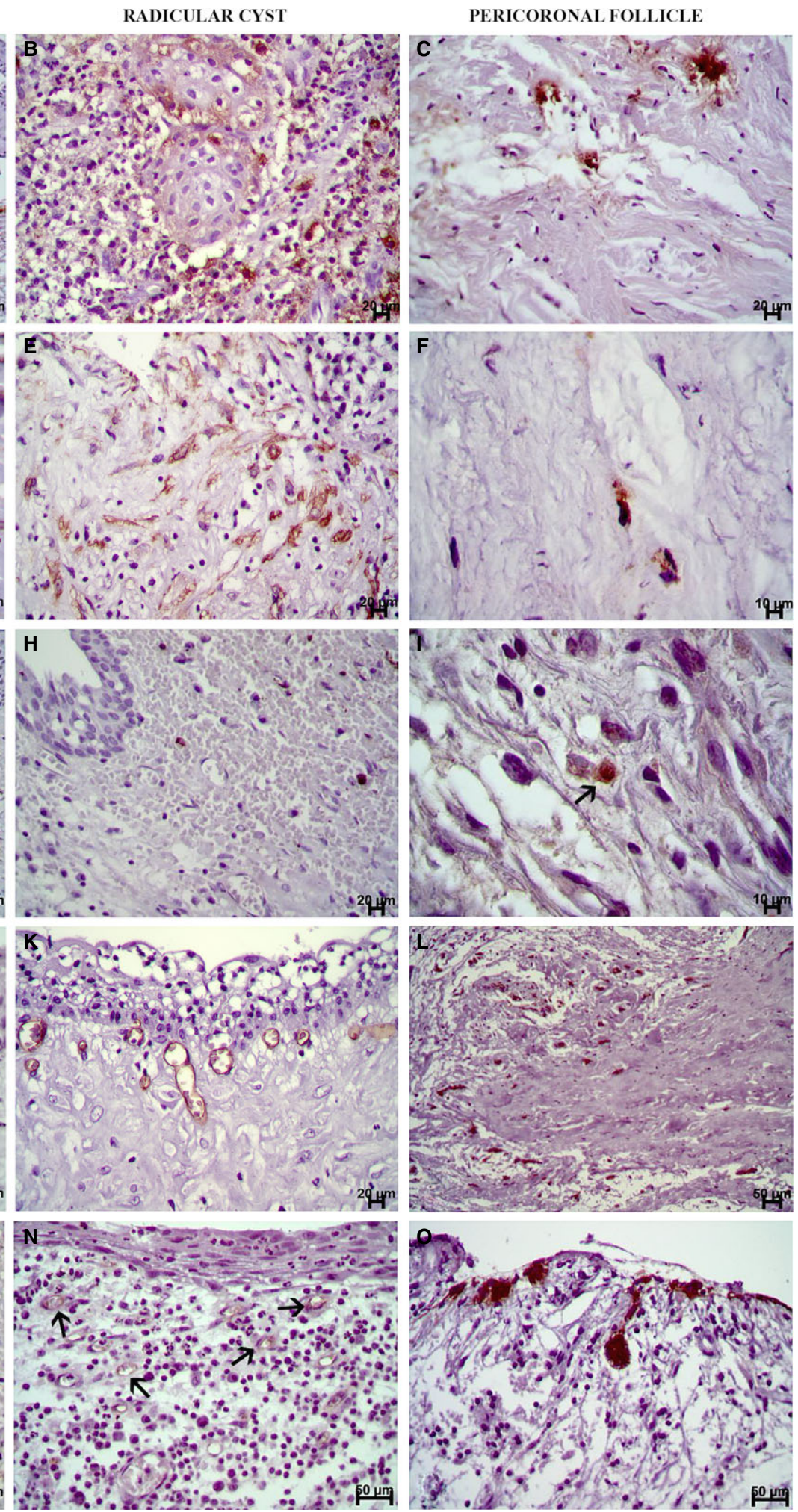

Figure 1 MAST CELLS (EnVision ${ }^{\mathrm{TM}}$ polymeric system) (A) Degranulating mast cells scattered throughout cystic fibrous wall. (B) High number of mast cells scattered throughout cystic fibrous wall that shows marked chronic inflammation. (C) Note degranulating mast cells in pericoronal follicle. MYOFIBROBLASTS (EnVision ${ }^{\mathrm{TM}}$ polymeric system): (D) Note a high number of myofibroblasts seen along the fibrous cystic wall; they are located parallel or not to the epithelial lining. (E) Note different shapes of myofibroblasts present in area. (F) Note rare myofibroblasts present in this area. MACROPHAGES (EnVision $^{\mathrm{TM}}$ polymeric system): (G) CD163-positive macrophages scattered throughout fibrous cystic wall. (H) Rare CD163-positive macrophages are seen in the in the fibrous wall of the cyst. (I) Only one CD163-positive macrophage is seen in the fibrous wall (arrow). ANGIOGENESIS (EnVision ${ }^{\mathrm{TM}}$ polymeric system): (J) CD34-positive blood vessels located close to the epithelial lining. (K) CD34-positive blood vessels present in the granulation tissue. (L) Note small CD34-positive blood vessels. LYMPHANGIOGENESIS (EnVision ${ }^{\mathrm{TM}}$ polymeric system): (M) Small D2-40-positive lymphatic vessels in the cystic wall. (N) Small D2-40-positive lymphatic vessels in granulation tissue (arrows). (O) Odontogenic epithelial remnants are positives for D2-40. 

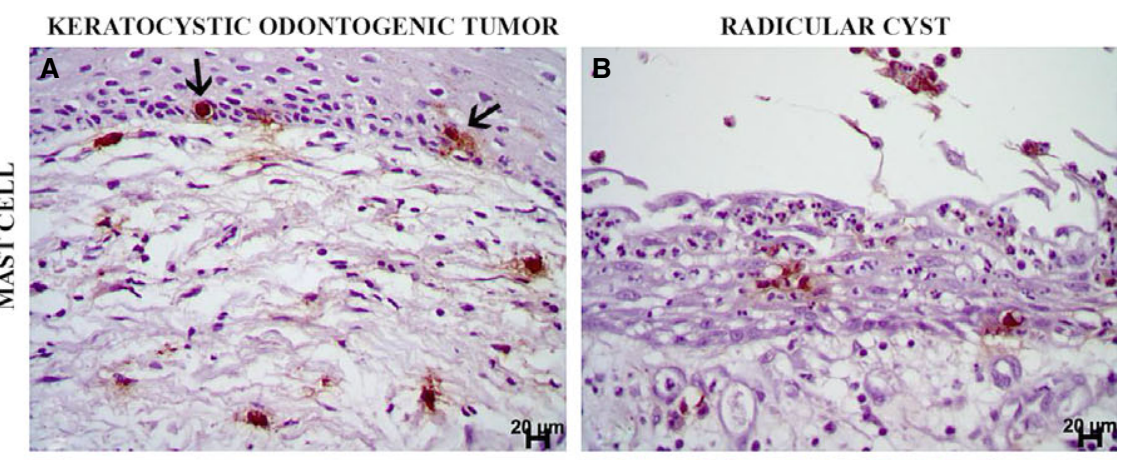

Figure 2 : MAST CELLS (EnVision $^{\mathrm{TM}}$ polymeric system): (A) Note mast cells with different shapes that located in the basal cell and intermediate layer of cystic lining and along the cystic wall. (B) Note mast cells entrapped in the basal cell and intermediate layer of cystic lining as well as in the lumen. MYOFIBROBLASTS(EnVision ${ }^{\text {TM }}$ polymeric system): (C) Different shapes of myofibroblasts are present in area with granulation tissue. (D) Bulky myofibroblast close to epithelial lining. MACROPHAGES(EnVision ${ }^{\mathrm{TM}}$ polymeric system): (E) A rare CD163-positive macrophage is seen in the epithelial lining (arrow). LYMPHANGIOGENESIS (EnVision ${ }^{\mathrm{TM}}$ polymeric system): (F) Epithelial buddings are positives for D2-40 particulary in basal and parabasal cells.
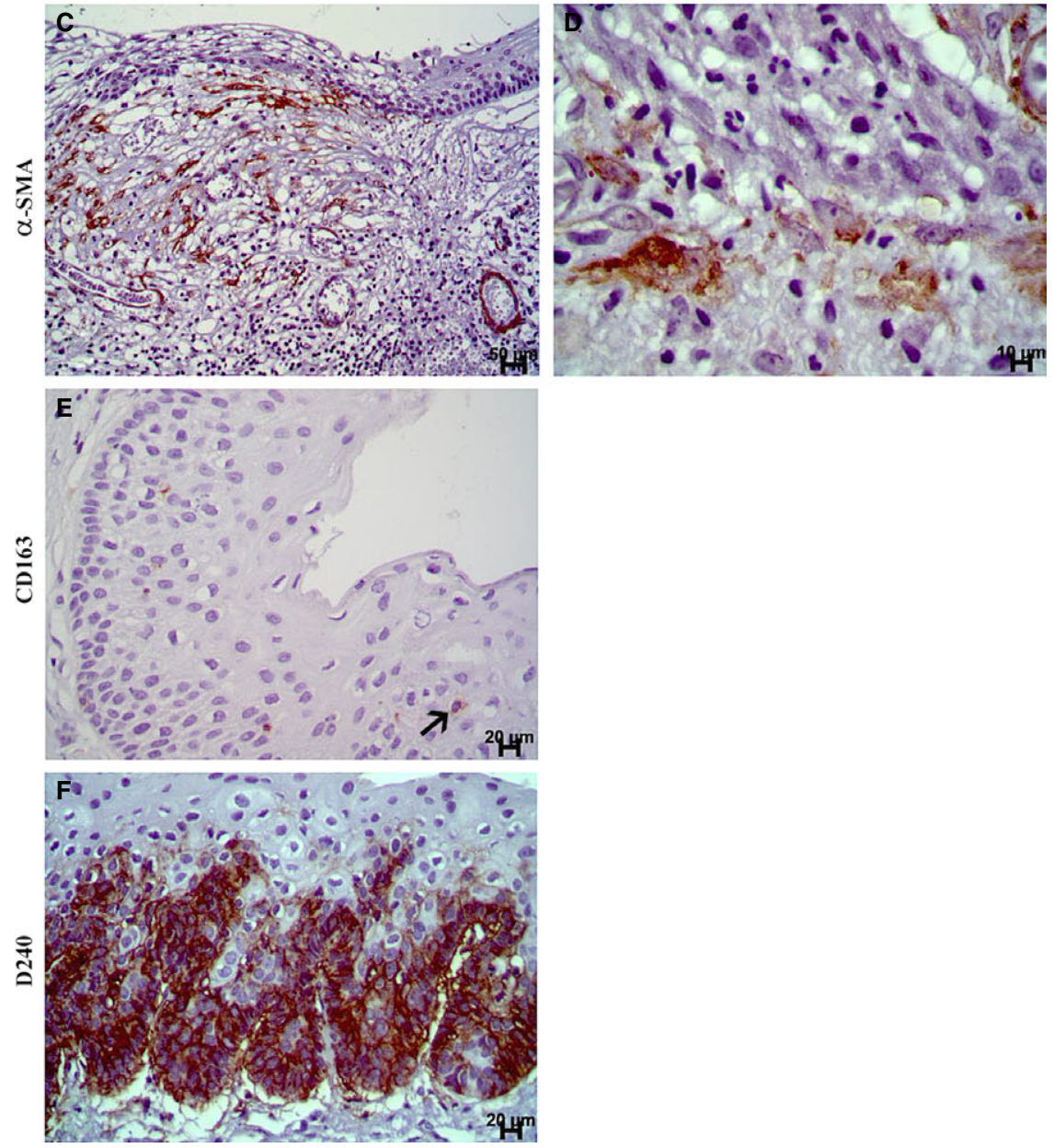

(Fig. 1K) and were concentrated near to the inflammatory infiltrate in most cases. In RCs, mean angiogenic indices ranged from 4.68 to 25.53 (mean $14.09 \pm 5.83$ ). In PFs, CD34-positive blood vessels were detected in $100 \%$ of cases (Fig. 1L).

There was no statistically significant difference in MVD between KCOTs, PFs, and RCs $(P=0.244$, one-way ANOVA). A summary of results is seen in Table 2 .

\section{Immunoexpression of D2-40}

D2-40-positive lymphatic vessels were detected in $96.6 \%$ $(n=29)$ of the KCOT specimens analyzed (Fig. 1M) and they were increased in relation to RCs and PFs. They were evident at different sites in the cystic wall. Mean values ranged from 0 to 28.29 (mean of $11.64 \pm 6.56$ ). Strong expression of D2-40 was observed in the cell membrane and in the cytoplasm of epithelial cells of the basal and suprabasal layers in $100 \%$ of the KCOT cases analyzed $(n=30$, Fig. 2F).

Although scarce, D2-40 immunostaining was observed in 93.3\% $(n=14)$ of the RC cases analyzed, especially in granulation tissue (Fig. 1N). Variable expression of podoplanin was detected in the cell membrane and cytoplasm of epithelial cells. The lymphangiogenic indices in RCs ranged from 0 to 5.31 (mean $4.19 \pm 1.253$ ).

PFs showed D2-40 immunoexpression in only $57.14 \%$ $(n=4)$ of cases, and lymphangiogenic indices in PFS ranged from 0 to 0.55 (mean $0.167 \pm 0.224$ ). In some 
Table 2 Mean density $/ \mathrm{mm}^{2}$ of mast cells, myofibroblasts, macrophages, microvessels, and lymphatic microvessels between keratocystic odontogenic tumors, radicular cysts, and pericoronal follicles

\begin{tabular}{lrccc}
\hline Variable & $\mathrm{n}$ & Mean & SD & P-value* \\
\hline Mast cells & & & & \\
KCOT & 30 & 7.398 & 1.653 & $0.00^{* *}$ \\
RC & 15 & 22.7 & 8.15 & $0.00^{* *}$ \\
PF & 7 & 1.234 & 2.072 & \\
Myofibroblasts & & & & \\
KCOT & 30 & 29.25 & 15.18 & $0.00^{* *}$ \\
RC & 15 & 4.663 & 2.6 & \\
PF & 7 & 1.5 & 0.445 & \\
Macrophages & & & & \\
KCOT & 30 & 2.31 & 2.921 & 0.084 \\
RC & 15 & 1.423 & 1.362 & \\
PF & 7 & 0.143 & 0.151 & \\
Angiogenesis & & & & \\
KCOT & 30 & 16.58 & 5.98 & 0.244 \\
RC & 15 & 14.09 & 5.83 & \\
PF & 7 & 13.32 & 4.85 & \\
Lymphangiogenesis & & & \\
KCOT & 30 & 11.64 & 6.56 & $0.00^{* *}$ \\
RC & 15 & 4.19 & 1.253 & \\
PF & 7 & 0.167 & 0.224 & \\
\hline
\end{tabular}

*One-way ANOVA. KCOT: keratocystic odontogenic tumor; RC: radicular cyst; PF: pericoronal follicle; SD: standard deviation. ${ }^{* *}$ Statistically significant.

specimens, staining occurred in epithelial remnants, if present (Fig. 1O).

A significant difference in D2-40-positive vessels was observed for KCOTs when compared to RCs and PFs $(P<0.01$, one-way ANOVA $)$ and difference between RCs and PFs was not statistically significant $(P>0.05)$. A summary of results is seen in Table 2 .

\section{Inflammatory infiltrate}

Significant inflammation was observed in $33.3 \%(n=10)$ of KCOT cases, and non-significant inflammation in $66.67 \%$ $(n=20)$. In contrast, significant inflammation was detected in all cases of RCs. The Student's $t$-test revealed no significant association between markers and the presence of inflammation $\left(\mathrm{P}_{\text {mast cells } \mathrm{x} \text { inflammation }}=0.031 ; \mathrm{P}_{\mathrm{SMA} \times \text { inflam- }}\right.$ mation $=0.401 ; \mathrm{P}_{\mathrm{CD} 163 \times \text { inflammation }}=0.276 ; \mathrm{P}_{\mathrm{CD} 34} \mathrm{x}$ inflammation $\left.=0.528 ; \mathrm{P}_{\mathrm{D} 2-40 \times \text { inflammation }}=0.148\right)$.

Correlation between stromal markers in the lesions studied Analysis between mast cell tryptase and CD34 in KCOTs indicated a moderate positive correlation $(P=0.025$, $r=0.409$ ). No correlation was observed between the other markers.

\section{Radicular cysts}

Analysis between $\alpha$-SMA and CD34 revealed a significant moderate inverse correlation $(P=0.017, \quad r=-0.603)$. There was no correlation between the other markers.

\section{Pericoronal follicles}

Analysis between $\alpha$-SMA and CD34 showed a strong inverse correlation $(P=0.049, r=-0.756)$. No correlation was observed between the other markers.

\section{Discussion}

The results of this study showed a proportionally higher participation of MFs, CD163-positive macrophages, CD34-positive blood vessels, and D2-40-positive lymphatic vessels in KCOTs when compared to RCs and PFs. In contrast, the MC population was lower in KCOTs compared with RCs, but was substantially higher when compared to PFs.

In addition to being found in the proximity of blood vessels, MCs were also be present on the epithelial lining (29). This spatial distribution of MCs was also observed in the KCOT cases of the present study, in agreement with other authors $(4,24,26)$ and has also been reported for RCs (24), and dentigerous cysts $(24,26)$. However, the density of these cells was higher in RCs, followed by KCOTs and $\mathrm{PFs}$, and this difference was statistically significant. In contrast, Chatterjee et al. (26) found a higher density of $\mathrm{MCs} / \mathrm{mm}^{2}$ in KCOTs and a lower density in RCs, but this could be due to histochemistry for Toluidine blue. It is possible that this cell population prevails, considering maintenance of the fibrous wall of these lesions, and actively participates in tissue remodeling as proposed by Pereira et al. (4) and Conceição et al. (35).

Although Rodini et al. (25) have attributed the presence and distribution of MCs in cystic lesions to attraction by peripheral areas of active inflammation, in the present study most KCOTs did not exhibit significant inflammation, but contained a considerable density of MCs. However, we also found areas with significant inflammation and a considerable number of MCs in KCOTs and RCs. In contrast to KCOT, we observed MCs in the cystic lumen of RCs, as also reported in a previous study (25).

The transdifferentiation of fibroblasts into MFs plays a critical role in the progression and development of tumors (12). According to Vered et al. (13), Nonaka et al. (15), MFs are found in ameloblastomas, KCOTs, and odontogenic cysts and may be related to the aggressive behavior of these lesions (13). In the present study, the population of MFs was detected in all cases of KCOT, in agreement with Nonaka et al. (15) and Ramos et al. (16). Furthermore, the number of MFs was significantly higher in KCOTs compared with RCs and PFs. Nadalin et al. (14) also found a larger number of MFs in KCOTs when compared to radicular and dentigerous cysts. These findings suggest that MFs also contribute to the growth and expansion of KCOTs and, similarly, are involved in the early development of RCs. According to Vered et al. (13), an increase in MFs in these lesions could be a result of increased expression of TGF- $\beta$ in the epithelium of odontogenic lesions, a fact that would explain the aggressive behavior of KCOTs and ameloblastomas.

Although no significant correlation was observed in the present study between the MC population and MFs, a large number of both cells was detected in the KCOT and so was a large number of MCs detected in the granulation tissue of KCOTs, which may contribute to the growth of these tumors through the release of proteases and extracellular matrix remodeling. In view of these findings, it is possible to hypothesize that tryptase released by MCs stimulates the 
proliferation of fibroblasts and the synthesis of collagen, altering the tumor microenvironment, which becomes denser and more contractile, as it facilitates the expression of $\alpha$-SMA by fibroblasts and their transdifferentiation into MFs $(8,12)$. In this respect, other hypotheses can be raised to explain these events. In an in vitro study culturing $\mathrm{MCs}$ together with fibroblasts, Frungieri et al. (36) observed that MCs can stimulate the expression of COX-2 and production of PGE2, thus inducing fibroblast proliferation and the synthesis of type I collagen. In the present study, no correlation was observed between MCs and MFs, but a larger number of MCs was associated with areas of higher MVD.

CD163 is a marker expressed by macrophages with the M2 phenotype (17). In the present study, CD163-positive macrophages were detected in KCOTs, RCs, and PFs. However, we found no significant differences in the mean density of macrophages between these lesions and PFs. In KCOTs, macrophages were observed near the parenchyma and especially close to areas of inflammation. The latter is not a surprising finding since macrophages can be recruited in response to different inflammatory mediators $(35,37)$. To our knowledge, this is the first study reporting the presence of CD163-positive macrophages in KCOT. Other authors such as Guzmán-Medrano et al. (34) found a relatively large number of macrophages in ameloblastomas, which was considerably higher than that observed in the KCOT, RC, and PF cases studied here. This difference may be related to differences in the methods employed since these authors used CD68 as a marker.

We also found no correlation between the density of MCs and CD163-positive cells in KCOTs, RCs, and PDs, although CD163-positive macrophages were detected in all lesions. Furthermore, MCs and macrophages shared similar regions in KCOTs, including the area beneath the epithelial lining and deep regions of the fibrous capsule.

No correlation between the CD163 and $\alpha$-SMA markers was found in the present study. However, as observed for MCs and MFs, macrophages were more frequent in KCOTs and RCs than in PFs, suggesting that these stromal components somehow contribute to the pathogenesis of KCOT and RC.

The CD34 marker in the lesions studied showed a slightly higher MVD in KCOTs compared with RCs and PFs, but without significant differences, demonstrating strong migration and vascular mobilization during the development of these lesions and PFs. The blood vessels were mainly located near the epithelium of KCOT, as also reported by Nonaka et al. (15) and Alaeddini et al. (30). Large concentrations of blood vessels were not only detected in areas of significant inflammation, but also in areas where significant inflammation was absent.

CD34 immunostaining in KCOTs and RCs compared with PFs showed a higher, although discrete, MVD in the former. Similarly, in a study on angiogenesis in benign and malignant ameloblastomas using the same marker, Kumamoto et al. (31) observed a higher MVD in these lesions compared with tooth germs. These results suggest that aggressive lesions require a larger number of blood vessels in these lesions and greater tumor growth.
Interestingly, a moderate positive correlation was observed between MCs and CD34 in KCOTs, demonstrating that the presence of blood vessels is accompanied by the presence of MCs and vice versa. These findings thus suggest that MCs contribute to blood vessel formation as previously reported. According to Ribatti and Crivellato et al. (5), the proangiogenic property of $\mathrm{MCs}$ is attributed to their capacity of secreting molecules such as VEGF, FGF-2, IL-8, and TGF- $\beta$.

Myofibroblasts are an important source of VEGF and cyclooxygenase 2, two well-known proangiogenic factors (37). In the present study, we found no correlation between the presence of MFs and the angiogenic index in KCOTs, in agreement with the results of Nonaka et al. (15). However, curiously, a moderate inverse correlation between the presence of MFs and the angiogenic index was observed in RCs and a strong inverse correlation in PFs. These results could suggest that, in contrast to RCs and PFs, MFs are not directly involved in vessel formation in KCOTs.

There was also no correlation between CD163 and CD34 between KOTs and RCs, although macrophages secrete VEGF, thus contributing to angiogenesis (37). We believe that these biomarkers are involved in the formation of KCOTs and RCs. In contrast, a positive correlation between angiogenesis and macrophage count has been demonstrated in different tumors such as breast cancer (18).

As observed for angiogenesis, lymphangiogenesis is induced by growth factors that are secreted by cells such as macrophages and MCs (22). The expression of D2-40 in KCOTs showed strong immunoexpression of this marker in the cell membrane and cytoplasm of epithelial cells of the basal and suprabasal layers. Okamoto et al. (33) associated this strong expression of podoplanin with basal cell proliferative activity and the formation of epithelial islands and suggested that this protein plays a role in the local invasiveness and progression of tumors such as KCOT. In the present study, lymphatic vessels were detected in almost $100 \%$ of the KCOT specimens analyzed and were more evident at sites distant from the epithelium. The lymphangiogenic index was significantly higher in KCOTs than in RCs and PFs. These findings suggest that podoplanin is related to a more neoplastic nature rather than the development itself of RC and PF, although the lymphangiogenic index was significantly higher in RCs than in PFs.

According to Jackson et al. (28), MFs promote lymphangiogenesis in vitro by secreting a series of cytokines, including VEGF. In the present study, no correlation was observed between MFs and D2-40-positive vessels in the different lesions and PFs. Further studies are necessary to clarify this matter.

In conclusion, this study demonstrated a significant interaction between the MC population and CD34-positive vessels in KCOTs, as well as an inverse relationship between MFs and CD34-positive vessels in RCs and PFs, supporting the hypothesis that MCs and blood vessels contribute to the stromal scaffold of KCOT. Although we found no interaction between the other cellular and vascular components studied, they probably contribute to the pathogenesis particularly of KCOT and RC. 


\section{References}

1. Philipsen HP. Keratocystic odontogenic tumor. In: Eveson JW, Reichart P, Sidransky D, eds. Barnes L. World Health Organization classification of tumors. Pathology and genetics of head and neck tumors. Lyon: IARC Press, 2005; 306-7.

2. Gomes CC, Diniz MG, Gomez RS. Review of the molecular pathogenesis of the odontogenic keratocyst. Oral Oncol 2009; 45: 1011-4.

3. Mello LA, Gurgel CA, Ramos EAG, et al. Keratocyst odontogenic tumour: An experience in the Northeast of Brazil. Srp Arh Celok Lek 2011; 139: 291-7.

4. de Assis Caldas Pereira F, Gurgel CA, Ramos EA, et al. Distribution of mast cells in benign odontogenic tumors. Tumour Biol 2012; 33: 455-61.

5. Ribatti D, Crivellato E. "Sprouting angiogenesis", a reappraisal. Dev Biol 2012; 372: 157-65.

6. Caughey GH. Mast cell tryptases and chymases in inflammation and host defense. Immunol Rev 2007; 217: 141-54.

7. Stone K, Prussin C, Metcalfe D. IgE, Mast Cells, Basophils and Eosinophils. J Allergy Clin Immunol 2010; 125: S73-80.

8. Desmouliere A, Chaponnier C, Gabbiani G. Tissue repair, contraction, and the myofibroblast. Wound Repair Regen 2005; 13: $7-12$.

9. De Castro IC, Rocha CA, Gomes Henriques AC, et al. Do Laser and led phototherapies influence mast cells and myofibroblasts to produce collagen? Lasers Med Sci 2014; 29: 1405-10.

10. Stockmann C, Schadendorf D, Klose R, Helfrich I. The impact of the immune system on tumor: angiogenesis and vascular remodeling. Front Oncol 2014; 4: 69.

11. Desmoulière A, Guyot C, Gabbiani G. The stroma reaction myofibroblast: a key player in the control of tumor cell behavior. Int J Dev Biol 2004; 48: 509-17.

12. Mangia A, Malfettone A, Rossi R, et al. Tissue remodelling in breast cancer: human mast cell tryptase as an initiator of myofibroblast differentiation. Histopathol 2011; 58: 1096-106.

13. Vered M, Shoha I, Buchner A, Dayan D. Myofibroblasts in stroma of odontogenic cysts and tumors can contribute to variations in the biological behavior of lesions. Oral Oncol 2005; 41: 1028-33.

14. Nadalin MR, Fregnani ER, Silva-Sousa YT, Da Cruz Perez DE. Presence of myofibroblasts and matrix metalloproteinase 2 in radicular cysts, dentigerous cysts, and keratocystic odontogenic tumors: a comparative immunohistochemical study. J Endod 2012; 38: 1363-7.

15. Nonaka CF, Cavalcante RB, Nogueira RL, de Souza LB, Pinto LP. Immunohistochemical analysis of bone resorption regulators (RANKL and OPG), angiogenic index, and myofibroblasts in syndrome and non-syndrome odontogenic keratocysts. Arch Oral Biol 2012; 57: 230-7.

16. De Oliveira Ramos G, Costa A, Meurer MI, Vieira DS, Rivero ER. Immunohistochemical analysis of matrix metalloproteinases (1, 2, and 9), Ki-67, and myofibroblasts in keratocystic odontogenic tumors and pericoronal follicles. $J$ Oral Pathol Med 2014; 43: 282-8.

17. Barros MH, Hassan R, Niedobitek G. Tumor-associated macrophages in pediatric classical hodgkin lymphoma: association with epstein-barr virus, lymphocyte subsets, and prognostic impact. Clin Cancer Res 2012; 18: 3762-71.

18. Medrek C, Pontén F, Jirström K, Leandersson K. The presence of tumor associated macrophages in tumor stroma as a prognostic marker for breast cancer patients. BMC Cancer 2012; 12: 306 .

19. Herrera M, Herrera A, Domínguez G, et al. Cancer-associated fibroblast and M2 macrophage markers together predict outcome in colorectal cancer patients. Cancer Sci 2013; 104: 437-44.

20. Weidner N, Semple JP, Welch WR, Folkman J. Tumour angiogenesis and metastasis - correlation in invasive breast carcinoma. N Engl J Med 1991; 324: 1-8.

21. Folkman J, Kalluri R. Cancer without disease. Nature 2004 2004; 427: 787.

22. Mohammed RA, Green A, El-Shikh S, Paish EC, Ellis IO, Martin SG. Prognostic significance of vascular endothelial cell growth factors -A, -C and -D in breast cancer and their relationship with angio- and lymphangiogenesis. $\mathrm{Br}$ J Cancer 2007; 96: 1092-100.

23. Ucuzian AA, Gassman AA, East AT, Greisler HP. Molecular mediator of angiogenesis. J Burn. Care Res 2010; 31: 158-75.

24. De Noronha Santos Netto J, Pires FR, da Fonseca EC, Silva LE, De Queiroz Chaves Lourenço S. Evaluation of mast cells in periapical cysts, dentigerous cysts, and keratocystic odontogenic tumors. J Oral Pathol Med 2012; 41: 630-6.

25. Rodini CO, Batista AL, Lara VS. Comparative immunohistochemical study of the presence of mast cells in apical granulomas and periapical cysts: possible role of mast cells in the course of human periapical lesions. Oral Surg Oral Med Oral Pathol Oral Radiol Endod 2004; 97: 59-63.

26. Chatterjee S, Mahajan S, Boaz K, George T. Quantitative role of mast cells in odontogenic cystic enlargement. Braz J Oral Sci 2008; 7: 1662-5.

27. Maeda K, Chung YS, Takatsuka S, et al. Tumour angiogenesis and tumour cell proliferation as prognostic indicators in gastric carcinoma. Br J Cancer 1995; 72: 319-23.

28. Jackson DG. New molecular markers for the study of tumour lymphangiogenesis. Anticancer Res 2001; 21: 4279-83.

29. Ribatti D, Crivellato E, Molicac S. Mast cells and angiogenesis in haematological malignancies. Leuk Res 2009; 33: 876-9.

30. Alaeddini M, Salah S, Dehghan F, Eshghyar N, EtemadMoghadam S. Comparison of angiogenesis in keratocystic odontogenic tumours, dentigerous cysts and ameloblastomas. Oral Dis 2009; 15: 422-7.

31. Kumamoto H, Ohki K, Ooya K. Association between vascular endothelial growth factor (VEGF) expression and tumor angiogenesis in ameloblastomas. J Oral Pathol Med 2002; 31: 28-34.

32. Okamoto E, Kikuchi K, Miyazaki Y, et al. Significance of podoplanin expression in keratocystic odontogenic tumor. J Oral Pathol Med 2010; 39: 110-4.

33. Guzmán-Medrano R, Arreola-Rosales RL, Shibayama M, Silva-Olivares DA, Bologna-Molina R, Rodríguez MA. Tumor-associated macrophages and angiogenesis: a statistical correlation that could reflect a critical relationship in ameloblastoma. Pathol Res Pract 2012; 208: 672-6.

34. Conceição JG, Gurgel CA, Ramos EA, et al. Oral mucoceles: A clinical, histopathological and immunohistochemical study. Acta Histochem 2014; 116: 40-7.

35. Frungieri MB, Weidinger S, Meineke V, Kohn FM, Mayerhofer A. Proliferative action of mast-cell tryptase is mediated by PAR2, COX2, prostaglandins, and PPARgamma: possible relevance to human fibrotic disorders. Proc Natl Acad Sci USA 2002; 99: 15072-7.

36. Mosser DM, Edwards JP. Exploring the full spectrum of macrophage activation. Nat Rev Immunol 2008; 8: 958-67.

37. Konstantinopoulos PA, Vandoros GP, Karamouzis MV, Gkermpesi M, Sotiropoulou-Bonikou G, Papavassiliou AG. EGF-R is expressed and $\mathrm{AP}-1$ and $\mathrm{NF}-\kappa \mathrm{B}$ are activated in stromal myofibroblasts surrounding colon adenocarcinomas paralleling expression of COX-2 and VEGF. Cell Oncol 2007; 29: 477-82.

\section{Acknowledgement}

Authors thank FAPESB and CNPq for financial support. 